\title{
Resistência laríngea em indivíduos com fechamento velofaríngeo marginal
}

\author{
Laryngeal resistance in individuals with marginal \\ velopharyngeal closure
}

\author{
Carolina Macedo Battaiola Brustelloํ, Ana Paula Fukushiro², Renata Paciello Yamashita ${ }^{3}$
}

\begin{abstract}
RESUMO
Objetivo: Verificar se pacientes com disfunção velofaríngea marginal modificam a resistência laríngea como uma estratégia para alcançar o fechamento velofaríngeo completo durante a fala. Métodos: Foram avaliados 19 pacientes com fissura de palato operada, de ambos os sexos com idade entre 12 e 47 anos, com fechamento velofaríngeo marginal e 18 indivíduos sem fissura (grupo controle), de ambos os sexos, com idade entre 14 e 35 anos. A resistência laríngea (R), pressão aérea intra-oral $\left(\mathrm{P}_{\mathrm{o}}\right)$ e fluxo oro-nasal $(\stackrel{\circ}{\mathrm{V}})$ foram obtidos por meio de avaliação aerodinâmica utilizando-se o sistema PERCI-SARS durante a produção da sílaba /pa/, com e sem a oclusão das narinas. Resultados: Os valores médios de resistência laríngea (R), pressão aérea intra-oral $\left(\mathrm{P}_{\mathrm{o}}\right)$ e fluxo oronasal $(\stackrel{\circ}{V})$, no grupo com fechamento velofaríngeo marginal, foram de, respectivamente, $34,8 \pm 10,8 \mathrm{cmH}_{2} \mathrm{O} / \mathrm{L} / \mathrm{seg}, 4,8 \pm 1,4 \mathrm{cmH}_{2} \mathrm{O}$, $144,8 \pm 34,0 \mathrm{~mL} / \mathrm{s}$, sem a oclusão das narinas e de $34,0 \pm 14,3 \mathrm{cmH}_{2} \mathrm{O} / \mathrm{L} / \mathrm{seg}, 4,8 \pm 1,1 \mathrm{cmH}_{2} \mathrm{O}, 150,9 \pm 38,7 \mathrm{~mL} / \mathrm{s}$ com a oclusão das narinas. No grupo controle, os valores médios foram $39,2 \pm 13,4 \mathrm{cmH}_{2} \mathrm{O} / \mathrm{L} / \mathrm{seg}, 4,8 \pm 0,8 \mathrm{cmH}_{2} \mathrm{O}, 133,9 \pm 50,2 \mathrm{~mL} / \mathrm{s}$, respectivamente para as mesmas variáveis. Não houve diferença estatisticamente significante $(\mathrm{p}<0,05)$ entre os valores médios de resistência laríngea $(\mathrm{R})$, pressão aérea intra-oral $\left(\mathrm{P}_{\mathrm{o}}\right)$ e fluxo oro-nasal $(\mathrm{V})$ dos grupos estudados. Conclusão: Esses resultados mostraram que os pacientes com fechamento velofaríngeo marginal estudados não modificaram a resistência laríngea.
\end{abstract}

Descritores: Insuficiência velofaríngea; Fissura palatina; Laringe; Fala; Voz

\section{INTRODUÇÃO}

O funcionamento adequado do esfíncter velofaríngeo é fundamental para a produção normal da fala, uma vez que a ação coordenada dos músculos velofaríngeos direciona a corrente aérea expiratória para a cavidade oral e/ou nasal durante a fala $^{(1-3)}$. Nos pacientes com fissura labiopalatina, a anatomia e a fisiologia do esfíncter velofaríngeo estão alteradas e o objetivo

Trabalho realizado no Laboratório de Fisiologia do Hospital de Reabilitação de Anomalias Craniofaciais da Universidade de São Paulo - USP - Bauru (SP), Brasil.

(1) Pós-graduanda (Doutorado) do Programa de Pós-Graduação em Ciências da Reabilitação do Hospital de Reabilitação de Anomalias Craniofaciais da Universidade de São Paulo - USP - Bauru (SP), Brasil.

(2) Doutora, Professora do Departamento de Fonoaudiologia da Faculdade de Odontologia de Bauru da Universidade de São Paulo - USP - Bauru (SP), Brasil; Fonoaudióloga do Laboratório de Fisiologia do Hospital de Reabilitação de Anomalias Craniofaciais da Universidade de São Paulo USP - Bauru (SP), Brasil.

(3) Doutora, Fonoaudióloga do Setor de Fonoaudiologia e do Laboratório de Fisiologia do Hospital de Reabilitação de Anomalias Craniofaciais da Universidade de São Paulo - USP - Bauru (SP), Brasil.

Endereço para correspondência: Carolina Macedo Battaiola Brustello. R. Sílvio Marchione, 3-20, Vl. Universitária, Bauru (SP), Brasil, CEP: 17012900. E-mail: carolmb@usp.br

Recebido em: 21/1/2009; Aceito em: 6/5/2009 principal da cirurgia primária do palato é estabelecer condições anatômicas para a adequada função velofaríngea ${ }^{(4-6)}$. Entretanto, mesmo após a palatoplastia primária, alguns pacientes podem permanecer com a função velofaríngea inadequada e apresentar alterações de fala, tais como: hipernasalidade, emissão de ar nasal (audível ou não), fraca pressão intra-oral e distúrbios articulatórios compensatórios, que caracterizam a disfunção velofaríngea (DVF) ${ }^{(7-8)}$.

A disfunção velofaríngea pode variar quanto à sua gravidade, o que repercute na qualidade da fala. Quando, durante a fala, ocorre um toque inconsistente do véu do palato nas demais estruturas da velofaringe, o fechamento velofaríngeo é definido como do tipo marginal ${ }^{(3)}$. Nestes casos, pode ocorrer hipernasalidade leve e emissão de ar nasal, pois, apesar do fechamento velofaríngeo marginal ser suficiente para a geração de pressão aérea intra-oral em níveis adequados para a fala, é insuficiente para evitar a hipernasalidade e a emissão de ar nasal. Em alguns casos, ainda, a ressonância pode estar equilibrada, sendo o ronco nasal a única manifestação de fala característica da disfunção velofaríngea marginal. Estudos utilizando a nasofaringoscopia e videofluoroscopia demonstraram haver certa variabilidade no funcionamento do esfíncter velofaríngeo em indivíduos com função velofaríngea marginal. Em alguns casos, o fechamento velofaríngeo é alcançado, 
porém não se mantém por tempo suficiente e com a força necessária para evitar a emissão nasal de ar durante a produção das consoantes de pressão. Em outros, é possível ocorrer o fechamento velofaríngeo adequado durante a produção de algumas consoantes, porém na produção de vogais e semivogais o fechamento velofaríngeo pode ser incompleto ${ }^{(3,9)}$. Em todos esses casos o fechamento velofaríngeo pode ser conseguido com o aumento do esforço respiratório e consequentemente o aumento da pressão aérea intra-oral. Estas estratégias aerodinâmicas fazem parte de um sistema de regulação e controle que visa estabilizar a pressão aérea durante a fala a fim de compensar o funcionamento inadequado da velofaringe ${ }^{(10)}$. Entretanto, este esforço aumentado na produção da fala é difícil de ser mantido por um período prolongado, de tal forma que o fechamento velofaríngeo pode ser completo em algumas palavras ou sentenças, mas é perdido na fala encadeada ou em contextos de fala mais complexos. Um exemplo é o que ocorre com alguns pacientes quando a fala está adequada no início do dia e piora gradativamente, aumentando a hipernasalidade na medida em que o indivíduo se torna mais cansado ${ }^{(11)}$.

$\mathrm{O}$ fato de indivíduos com disfunção velofaríngea marginal conseguirem o fechamento velofaríngeo adequado durante a produção de fala e após certo tempo perderem este fechamento, passando a apresentar hipernasalidade, pode ser explicado pela fadiga do músculo levantador do véu palatino. Exames eletromiográficos comprovaram que, em indivíduos com hipernasalidade leve, a atividade do músculo levantador do palato é maior do que em indivíduos sem fissura, o que pode justificar a ocorrência da fadiga e consequentemente a piora na qualidade vocal ${ }^{(12)}$. A fadiga muscular pode ser a causa do declínio da força e do nível de atividade muscular durante a fala espontânea, nos indivíduos com fechamento velofaríngeo marginal ${ }^{(12-13)}$.

Além das condições musculares do mecanismo velofaríngeo, a produção da fala normal requer um controle preciso da pressão e do fluxo aéreo ao longo do trato respiratório e vocal. Uma vez que uma das funções da laringe é regular o fluxo expiratório, esta se torna uma das mais importantes válvulas no controle da aerodinâmica da fala na presença da disfunção velofaríngea. Métodos instrumentais aerodinâmicos permitem obter informações sobre a fisiopatologia da função laríngea durante a fala ${ }^{(14)}$. Um dos procedimentos mais apropriados para este objetivo é a medida da resistência oferecida pela laringe à passagem do ar que, em última análise, é a avaliação da resistência laríngea. Nesse método, a dinâmica laríngea é avaliada por meio da medida simultânea dos fluxos e pressões aéreos gerados no trato vocal, sendo um método indicado para aferir a função laríngea e identificar possíveis desvios, como os que parecem resultar do mau funcionamento do esfíncter velofaríngeo. A medida da resistência laríngea tem sido utilizada para investigar o comportamento da laringe na presença da disfunção velofaríngea ${ }^{(15-19)}$.

Tem-se buscado esclarecer a inter-relação entre o funcionamento do esfíncter velofaríngeo e da válvula laríngea durante a fala. Entretanto, nos casos em que os pacientes apresentam deficiências mínimas do mecanismo velofaríngeo, como o que ocorre na disfunção velofaríngea marginal, a literatura tem se mostrado escassa. Deste modo, o presente estudo foi realizado com o propósito de confirmar a hipótese de que os indivíduos que apresentam fechamento velofaríngeo marginal aumentam o esforço respiratório e vocal durante a fala e, consequentemente, a resistência laríngea, como uma manobra compensatória para alcançar o fechamento velofaríngeo completo, e assim, melhorar a nasalidade. Pretendeu-se ainda, verificar se a oclusão das narinas, condição que elimina o escape de ar pelo nariz, modifica a resistência laríngea nestes indivíduos.

\section{MÉTODOS}

O estudo foi desenvolvido no Laboratório de Fisiologia do Hospital de Reabilitação de Anomalias Craniofaciais da Universidade de São Paulo (HRAC-USP) e recebeu aprovação do Comitê de Ética e Pesquisa da instituição (Ofício nº 157/2007).

Participaram do estudo 19 indivíduos, de ambos os gêneros, sendo 12 homens e sete mulheres, com idade entre 12 e 47 anos (média de 22 \pm 11 anos), com fissura de palato associada ou não à fissura de lábio, corrigida cirurgicamente, e que apresentavam fechamento velofaríngeo marginal. Para fins de controle, participaram ainda do estudo, 19 indivíduos, de ambos os sexos, sem fissura labiopalatina, sendo nove homens e dez mulheres, com idade entre 14 e 35 anos (média de $21 \pm 5$ anos) sem manifestações vocais.

Os pacientes foram selecionados com base no resultado da avaliação nasofaringoscópica, realizada de rotina no HRAC-USP. Foram incluídos os pacientes que, segundo essa avaliação, apresentaram fechamento velofaríngeo marginal, ou seja, aqueles em que se observou contato inconsistente do véu palatino com as paredes da faringe, acompanhado ou não de borbulha de ar durante as emissões de pelo menos metade das 12 sentenças contendo fonemas orais plosivos e fricativos surdos e sonoros, utilizadas no protocolo de nasofaringoscopia do HRAC-USP.

Os pacientes com fechamento velofaríngeo marginal selecionados foram submetidos à avaliação perceptiva da fala ${ }^{(20)}$ com o objetivo de excluir aqueles com possíveis alterações vocais perceptíveis, fístula no palato de qualquer extensão ou distúrbios articulatórios compensatórios na emissão do fonema /p/, o qual foi utilizado para a medida da resistência laríngea. Para avaliar os aspectos vocais utilizou-se a escala GRBAS (Overall grade of roughness, breathiness, asteny, strain quality) ${ }^{(21)}$, a qual analisa os seguintes parâmetros: grau de alteração vocal, irregularidade nas vibrações das pregas vocais, soprosidade, fraqueza e tensão. Portanto, foram incluídos no estudo, em ambos os grupos, apenas os indivíduos que apresentavam escore igual a zero na escala GRBAS, indicando ausência de manifestações vocais.

\section{Medida da resistência laríngea}

A resistência laríngea foi medida por meio da técnica fluxopressão utilizando-se o sistema computadorizado PERCI-SARS (Microtronics Corporations, versão 3.30). No método descrito por Smitheran e Hixon ${ }^{(22)}$, a pressão subglótica é estimada pela medida da pressão oral durante a produção da consoante plosiva surda/p/, a qual apresenta características aerodinâmicas favoráveis. Durante a produção desta consoante, os lábios estão ocluídos, as pregas 
vocais estão em abdução, oferecendo pouca resistência glótica e a pressão ao longo do trato vocal é constante. No momento em que o fonema /p/ é produzido, os lábios se abrem e a pressão captada reflete a pressão abaixo da glote. O fluxo aéreo oro-nasal é medido durante a produção da vogal /a/ por meio de uma máscara facial de silicone colocada sobre a boca e o nariz do paciente e acoplada a um pneumotacógrafo aquecido conectado a um transdutor de pressão. Na produção da vogal /a/, os lábios estão abertos, as pregas vocais estão em adução, oferecendo maior resistência glótica à passagem do ar. O fluxo então captado pela máscara é o próprio fluxo de ar através da laringe. Já a pressão intra-oral é captada por meio de um cateter de polietileno de pequeno diâmetro inserido na máscara facial através de um orifício lateral e posicionado na cavidade oral do paciente; a outra extremidade é conectada a um transdutor de pressão diferencial. Os sinais dos transdutores são enviados ao sistema PERCI-SARS, sendo registrados e analisados por software específicos (Figura 1). O sinal de voz é captado por um microfone localizado a uma distância de $15 \mathrm{~cm}$ da boca do paciente, para mensuração da intensidade em nível de pressão sonora.

O indivíduo foi instruído a produzir uma sequência de sete sílabas "pa”, seguindo o modelo do examinador, usando intensidade de voz confortável e estável e em nível de pressão intra-oral habitual previamente determinado pelo examinador. Ao iniciar o exame, utilizando-se o próprio sistema PERCISARS, o indivíduo foi instruído a repetir a frase "Papai pintou a rampa" em intensidade de voz confortável e habitual. A pressão aérea intra-oral foi captada por meio de um cateter posicionado no interior da boca do paciente, e o valor obtido foi utilizado como referência para a medida da resistência laríngea. Dessa forma, durante toda a avaliação, a pressão oral foi, então, monitorada, tendo como base o valor de referência obtido pelo próprio paciente, de modo a assegurar que não ocorressem variações da pressão oral e, consequentemente, da intensidade vocal durante a medida da resistência laríngea.

Para o cálculo da resistência laríngea $(\mathrm{R})$ foram selecionadas três emissões, desprezando-se as duas primeiras e as duas últimas. A pressão subglótica utilizada para análise foi a obtida no pico da curva de pressão intra-oral da consoante plosiva /p/, selecionada automaticamente pelo sistema. O fluxo oro-nasal foi medido na emissão da vogal /a/, no ponto médio do traçado do fluxo (Figura 2). Uma vez que o método preconizado por Smitheran e Hixon ${ }^{(22)}$ determina que a avaliação da resistência laríngea em pacientes com DVF deve ser realizada com a oclusão das narinas a fim de se evitar o escape de fluxo aéreo pelo nariz, a medida da resistência laríngea foi igualmente realizada com a oclusão das narinas. Utilizou-se um obturador nasal (rolha) posicionado em cada narina do paciente para impedir o escape de ar nasal durante as emissões. Em função do correto funcionamento do mecanismo velofaríngeo, no grupo controle a medida da resistência laríngea com a oclusão das narinas foi desnecessária. A calibração do sistema foi feita diariamente com sinais conhecidos de fluxo (205 mL/s) e pressão $\left(6 \mathrm{cmH}_{2} \mathrm{O}\right)$, utilizando, respectivamente, um rotâmetro e um manômetro de água.

\section{Estatística}

Os valores de resistência laríngea $(\mathrm{R})$, pressão aérea intraoral $\left(\mathrm{P}_{\mathrm{o}}\right)$ e fluxo aéreo oro-nasal $(\mathrm{V})$ obtidos no grupo de pacientes com fechamento velofaríngeo marginal com e sem oclusão das narinas foram comparados com os valores obtidos no grupo controle utilizando-se o teste t de Student para amostras independentes ${ }^{(23)}$. Os valores de resistência laríngea $(\mathrm{R})$, pressão aérea intra-oral $\left(\mathrm{P}_{\mathrm{o}}\right)$ e fluxo aéreo oro-nasal (V) obtidos no grupo de pacientes com fechamento velofaríngeo marginal com a oclusão das narinas e sem a oclusão das narinas foram comparados utilizando-se o teste " $t$ " de Student para amostras pareadas ${ }^{(23)}$. Foram aceitos como significantes valores de $\mathrm{p}<0,05$.

\section{RESULTADOS}

As Tabelas 1 e 2 mostram os valores individuais, médios e desvio-padrão de $\mathrm{P}_{\mathrm{o}}, \stackrel{\mathrm{o}}{\mathrm{V}}$ e R obtidos nos grupos com fechamento velofaríngeo marginal e no grupo controle. Os valores médios

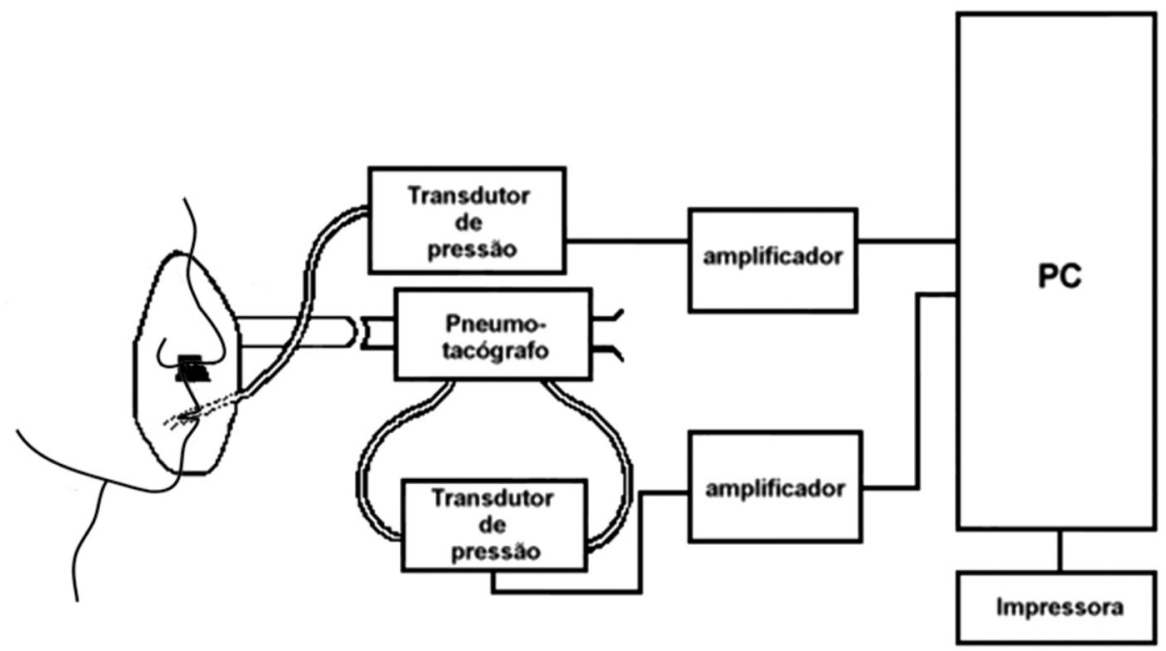

Figura 1. Sistema computadorizado PERCI-SAR (Microtronics Corporation) para medida da resistência laríngea. Adaptado de Trindade IE, Silva Filho MG. Fissuras labiopalatinas: uma abordagem interdisciplinar. São Paulo: Editora Santos; 2007. Diagnóstico instrumental da disfunção velofaríngea. p. 137. 


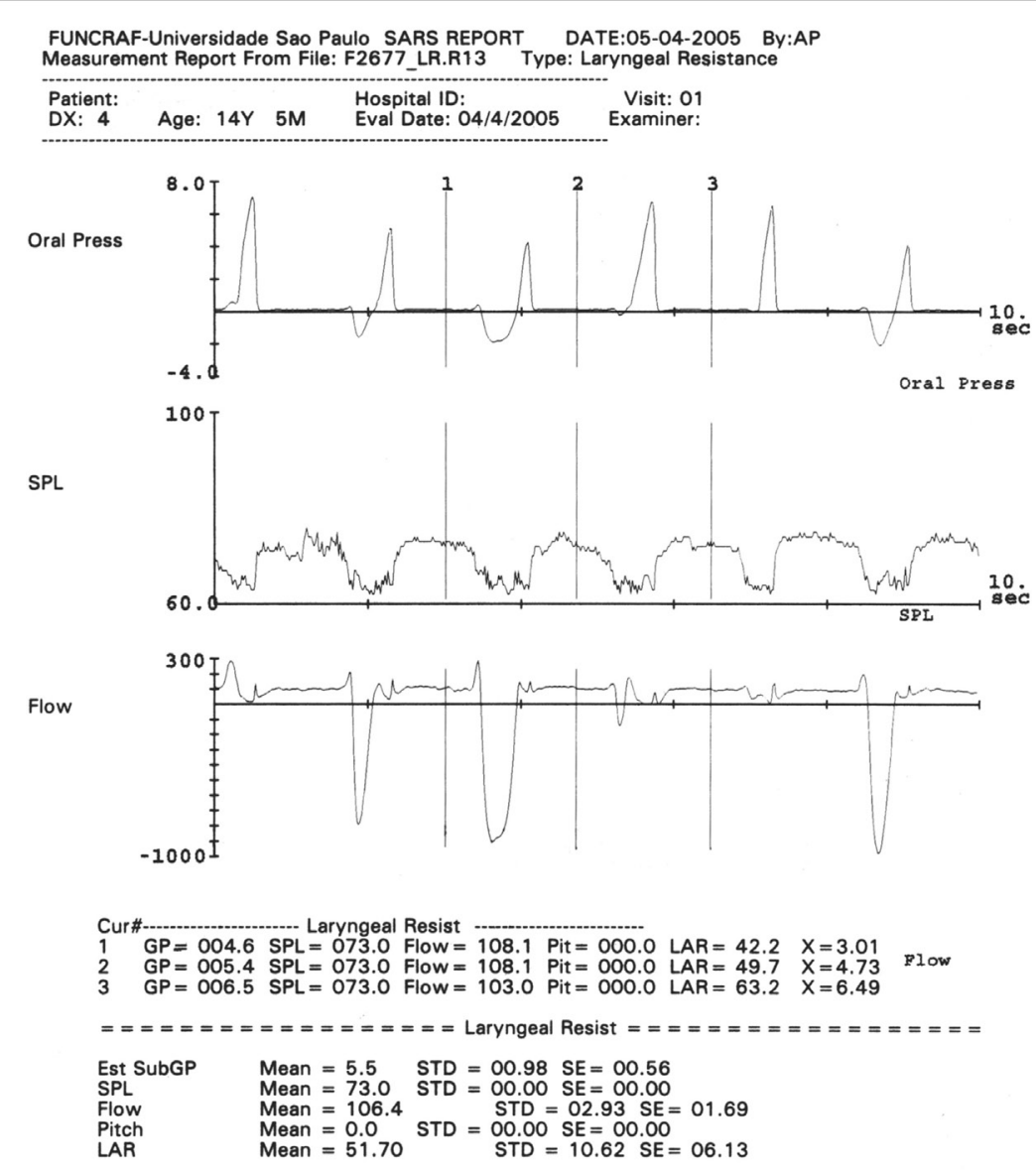

Figura 2. Traçado do registro da pressão oral, nível de intensidade sonora e fluxo oro-nasal para medida da resistência laríngea

Tabela 1. Valores individuais, médios e desvio padrão de pressão aérea intra-oral, fluxo aéreo laríngeo e resistência laríngea obtidos no grupo de pacientes com fechamento velofaríngeo marginal sem oclusão das narinas e com oclusão das narinas

\begin{tabular}{|c|c|c|c|c|c|c|}
\hline \multirow[t]{2}{*}{ Pacientes } & \multicolumn{3}{|c|}{ Ms } & \multicolumn{3}{|c|}{ Mc } \\
\hline & $\mathrm{P}_{0}\left(\mathrm{cmH}_{2} \mathrm{O}\right)$ & $\stackrel{\circ}{\mathrm{V}}(\mathrm{ml} / \mathrm{s})$ & $\mathrm{R}\left(\mathrm{cmH}_{2} \mathrm{O} / \mathrm{l} / \mathrm{s}\right)$ & $\mathrm{P}_{\mathrm{o}}\left(\mathrm{cmH}_{2} \mathrm{O}\right)$ & $\stackrel{\circ}{\mathrm{V}}(\mathrm{ml} / \mathrm{s})$ & $\mathrm{R}\left(\mathrm{cmH}_{2} \mathrm{O} / \mathrm{l} / \mathrm{s}\right)$ \\
\hline 1 & 3,7 & 165,9 & 22,79 & 3,4 & 199,5 & 16,83 \\
\hline 2 & 4,8 & 113,7 & 42,57 & 4,7 & 110,3 & 43,22 \\
\hline 3 & 5,2 & 100,0 & 52,02 & 4,9 & 108,0 & 45,88 \\
\hline 4 & 8,3 & 212,8 & 39,09 & 6,9 & 189,2 & 37,17 \\
\hline 5 & 5,5 & 106,4 & 51,70 & 6,2 & 79,4 & 79,22 \\
\hline 6 & 4,3 & 147,6 & 29,38 & 4,0 & 195,8 & 20,32 \\
\hline 7 & 3,8 & 184,7 & 20,45 & 4,1 & 168,7 & 23,96 \\
\hline 8 & 3,3 & 180,6 & 18,44 & 3,6 & 217,2 & 18,10 \\
\hline 9 & 3,8 & 122,6 & 31,60 & 4,6 & 134,2 & 34,29 \\
\hline 10 & 4,9 & 133,6 & 36,54 & 4,6 & 104,5 & 44,60 \\
\hline 11 & 3,1 & 134,5 & 23,54 & 2,7 & 111,0 & 24,49 \\
\hline 12 & 5,8 & 109,4 & 52,66 & 6,2 & 136,1 & 45,59 \\
\hline 13 & 3,3 & 150,0 & 21,78 & 5,0 & 141,9 & 24,66 \\
\hline 14 & 5,4 & 141,2 & 39,14 & 5,1 & 153,6 & 33,26 \\
\hline 15 & 4,6 & 113,4 & 40,78 & 4,1 & 134,6 & 31,24 \\
\hline 16 & 4,2 & 104,0 & 40,78 & 4,4 & 139,8 & 31,95 \\
\hline 17 & 5,0 & 163,9 & 30,32 & 4,6 & 168,5 & 27,22 \\
\hline 18 & 4,8 & 167,8 & 28,33 & 4,7 & 181,1 & 25,89 \\
\hline 19 & 7,8 & 198,6 & 39,50 & 7,0 & 193,5 & 37,29 \\
\hline $\bar{x}$ & 4,8 & 144,8 & 34,8 & 4,8 & 150,9 & 34,0 \\
\hline DP & 1,4 & 34,0 & 10,8 & 1,1 & 38,7 & 14,3 \\
\hline
\end{tabular}

Legenda: $\mathrm{Ms}$ = grupo de indivíduos com fechamento velofaríngeo marginal sem oclusão das narinas; $\mathrm{Mc}=$ grupo de indivíduos com fechamento velofaríngeo marginal com oclusão das narinas; $P_{0}=$ pressão aérea intra-oral; $\vee \stackrel{V}{=}$ fluxo aéreo oro-nasal; $R=$ resistência laríngea; $\bar{X}=$ média; $D P=$ desvio-padrão 
Tabela 2. Valores individuais, médios e desvio padrão de pressão aérea intra-oral, fluxo aéreo laríngeo e resistência laríngea dos indivíduos sem fissura (grupo controle)

\begin{tabular}{lccc}
\hline Sujeitos & $\mathrm{P}_{\mathrm{o}}\left(\mathrm{cmH}_{2} \mathrm{O}\right)$ & $\stackrel{\circ}{\mathrm{V}}(\mathrm{ml} / \mathrm{s})$ & $\mathrm{R}\left(\mathrm{cmH}_{2} \mathrm{O} / \mathrm{l} / \mathrm{s}\right)$ \\
\hline 1 & 4,5 & 72,4 & 63,11 \\
2 & 6,0 & 105,5 & 56,67 \\
3 & 5,0 & 117,2 & 42,63 \\
4 & 4,4 & 173,3 & 25,58 \\
5 & 5,4 & 139,5 & 38,59 \\
6 & 5,2 & 98,3 & 52,70 \\
7 & 4,3 & 211,4 & 20,46 \\
8 & 3,5 & 118,5 & 30,18 \\
9 & 6,6 & 277,8 & 23,78 \\
10 & 4,3 & 116,2 & 36,82 \\
11 & 5,1 & 76,7 & 66,09 \\
12 & 3,6 & 115,0 & 31,40 \\
13 & 4,1 & 124,0 & 33,18 \\
14 & 5,2 & 167,2 & 31,08 \\
15 & 4,2 & 88,6 & 47,65 \\
16 & 4,7 & 95,5 & 49,34 \\
17 & 4,1 & 155,5 & 26,37 \\
18 & 4,6 & 120,6 & 38,11 \\
19 & 5,5 & 171,4 & 31,92 \\
\hline $\bar{X}$ & 4,8 & 133,9 & 39,2 \\
\hline $\mathrm{DP}$ & 0,8 & 50,2 & 13,4 \\
\hline
\end{tabular}

Legenda: $\mathrm{Ms}$ = grupo de indivíduos com fechamento velofaríngeo marginal sem oclusão das narinas; $\mathrm{Mc}$ = grupo de indivíduos com fechamento velofaríngeo marginal com oclusão das narinas; $P_{0}=$ pressão aérea intra-oral; $\stackrel{\circ}{V}=$ fluxo aéreo oro-nasal; $\mathrm{R}=$ resistência laríngea; $\overline{\mathrm{X}}=$ média; $\mathrm{DP}=$ desvio-padrão

de $\mathrm{P}_{\mathrm{o}}$, ํำ e R obtidos no grupo com fechamento velofaríngeo marginal sem a oclusão das narinas foram, respectivamente, $4,8 \pm 1,4 \mathrm{cmH}_{2} \mathrm{O}, 144,8 \pm 34,0 \mathrm{~mL} / \mathrm{s}, 34,8 \pm 10,8 \mathrm{cmH}_{2} \mathrm{O} / \mathrm{L} / \mathrm{seg}$, e $4,8 \pm 1,1 \mathrm{cmH}_{2} \mathrm{O}, 150,9 \pm 38,7 \mathrm{~mL} / \mathrm{s}, 34,0 \pm 14,3 \mathrm{cmH}_{2} \mathrm{O} / \mathrm{L} / \mathrm{seg}$ com a oclusão das narinas. No grupo controle os valores foram $4,8 \pm 0,8 \mathrm{cmH}_{2} \mathrm{O}, 133,9 \pm 50,2 \mathrm{~mL} / \mathrm{s}, 39,2 \pm 13,4 \mathrm{cmH}_{2} \mathrm{O} / \mathrm{L} / \mathrm{seg}$, respectivamente.
Verificou-se que não houve diferença estatisticamente significante entre os valores obtidos com e sem a oclusão das narinas no grupo com fechamento velofaríngeo marginal. Verificou-se, ainda, que os pacientes com fechamento velofaríngeo marginal apresentaram uma tendência à resistência laríngea menor que a do grupo controle (Figuras 3 e 4). Entretanto, não foi encontrada diferença estatisticamente significante entre os valores obtidos em ambos os grupos (Tabelas 3, 4 e 5).

\section{DISCUSSÃO}

Considerando que a literatura sugere que indivíduos com disfunção velofaríngea podem aumentar o esforço respiratório durante a fala como uma manobra compensatória para alcançar o fechamento velofaríngeo adequado, o objetivo do presente estudo foi avaliar as repercussões aerodinâmicas deste esforço sobre a resistência laríngea de indivíduos com fechamento velofaríngeo marginal. Para tanto, foi utilizada uma abordagem aerodinâmica que fornece informações sobre o estado funcional da laringe, a avaliação da resistência laríngea obtida por meio da medida simultânea do fluxo aéreo oro-nasal e da pressão subglótica, durante a fala.

A investigação da relação entre a função velofaríngea e a função laríngea tem sido objeto de estudo há alguns anos, e os resultados relatados na literatura são bastante variados. Esta diversidade de achados decorre, provavelmente, da utilização de diferentes metodologias e, também, de variações nas características velofaríngeas dos grupos estudados. O que se pode observar nos estudos que avaliaram a função laríngea de pacientes com fissura palatina é que, em parte deles, os pacientes apresentavam fechamento velofaríngeo completo, ou seja, função velofaríngea adequada ${ }^{(15-16)}$ e em outra, apresentavam fechamento velofaríngeo inadequado indicativo de disfunção velofaríngea grave $\mathrm{e}^{(17-18)}$, o que pode justificar, portanto, resultados diferentes. Com base nos resultados obtidos nestes grupos distintos, a maioria dos autores enfatiza a necessidade de novos estudos a fim de investigar as características aerodinâmicas
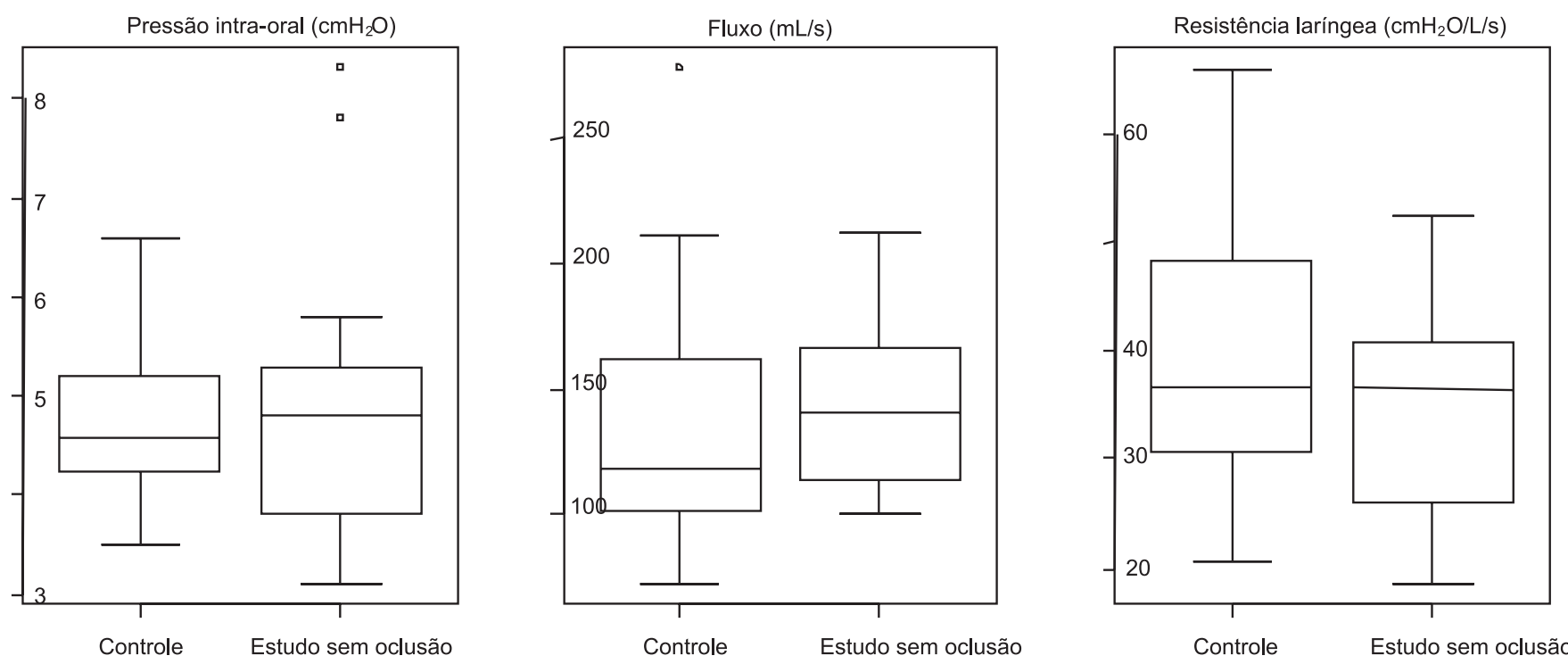

Figura 3. Boxplots referentes à distribuição dos valores de pressão aérea intra-oral, fluxo aéreo laríngeo e resistência laríngea do grupo controle e do grupo com fechamento velofaríngeo marginal sem oclusão das narinas 

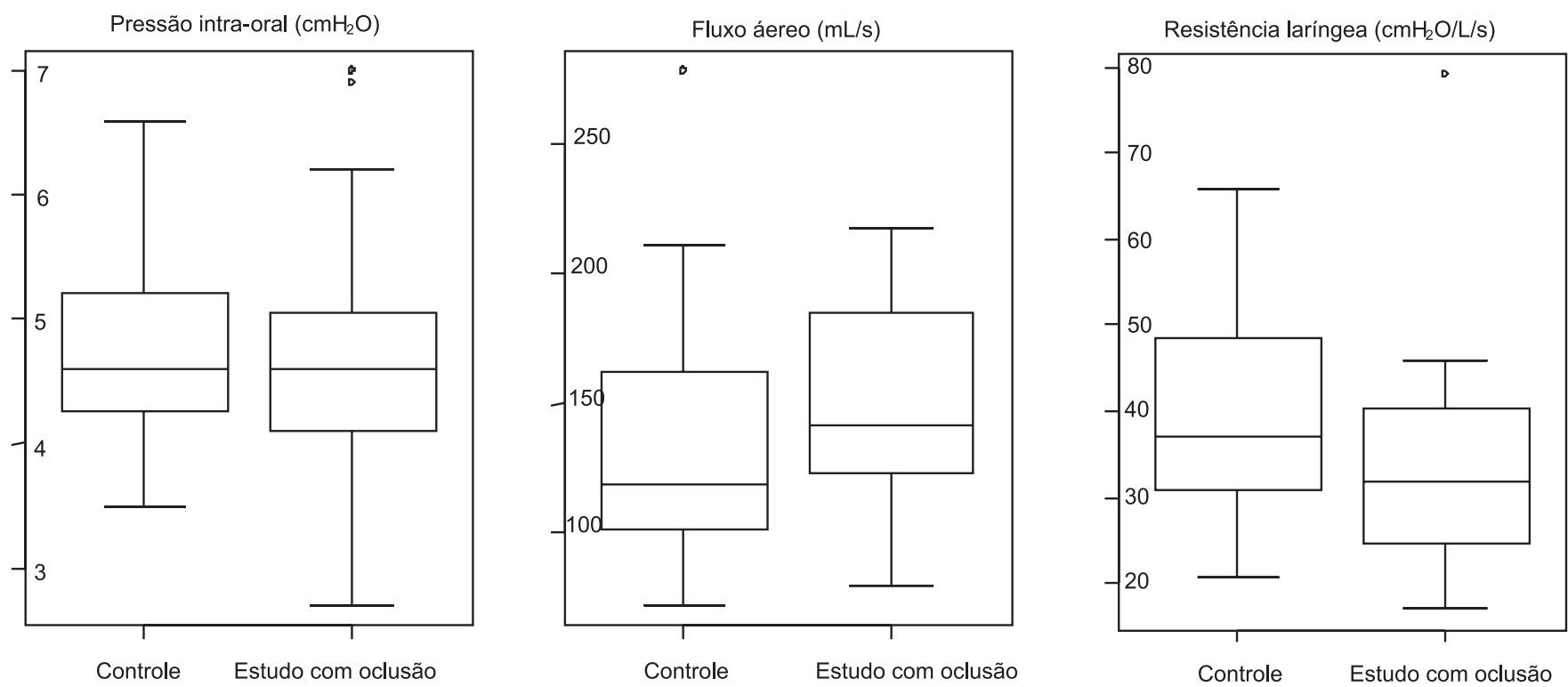

Figura 4. Boxplots referentes à distribuição dos valores de pressão aérea intra-oral, fluxo aéreo laríngeo e resistência laríngea do grupo controle e do grupo com fechamento velofaríngeo marginal com oclusão das narinas

Tabela 3. Comparação entre os valores médios e desvio-padrão de pressão aérea intra-oral, fluxo aéreo laríngeo e resistência laríngea com e sem a oclusão das narinas no grupo com fechamento velofaríngeo marginal

\begin{tabular}{|c|c|c|c|c|c|c|}
\hline \multirow[t]{2}{*}{ Variável } & \multicolumn{2}{|c|}{ Média \pm DP } & \multirow[t]{2}{*}{ Diferença média } & \multirow[t]{2}{*}{ Valor de $p$} & \multicolumn{2}{|c|}{ Intervalo de confiança (95\%) } \\
\hline & Sem oclusão & Com oclusão & & & Limite inferior & Limite superior \\
\hline$P_{0}$ & $4,8 \pm 1,4$ & $4,8 \pm 1,1$ & 0,0 & 0,786 & $-0,3$ & 0,4 \\
\hline$\stackrel{\circ}{\mathrm{V}}$ & $144,8 \pm 3,4$ & $150,9 \pm 38,7$ & $-6,1$ & 0,276 & $-17,5$ & 5,3 \\
\hline $\mathrm{R}$ & $34,8 \pm 10,8$ & $34,0 \pm 14,3$ & 0,9 & 0,663 & $-3,2$ & 4,9 \\
\hline
\end{tabular}

Teste $t$ de Student para amostras pareadas $(p<0,05)$

Legenda: $\mathrm{P}_{0}=$ pressão aérea intra-oral; $\stackrel{\circ}{V}=$ fluxo aéreo oro-nasal; $\mathrm{R}=$ resistência laríngea; $\mathrm{DP}=$ desvio-padrão

Tabela 4. Comparação entre os valores médios e desvio-padrão de pressão aérea intra-oral, fluxo aéreo laríngeo e resistência laríngea do grupo com fechamento velofaríngeo marginal sem a oclusão das narinas e do grupo controle

\begin{tabular}{|c|c|c|c|c|c|c|}
\hline \multirow[t]{2}{*}{ Variáveis } & \multicolumn{2}{|c|}{ Média \pm DP } & \multirow[t]{2}{*}{ Diferença média } & \multirow[t]{2}{*}{ Valor de $p$} & \multicolumn{2}{|c|}{ Intervalo de confiança (95\%) } \\
\hline & Controle & Sem oclusão & & & Limite inferior & Limite superior \\
\hline $\mathrm{P}_{0}$ & $4,8 \pm 0,8$ & $4,8 \pm 1,4$ & $-0,1$ & 0,853 & $-0,8$ & 0,7 \\
\hline V & $133,9 \pm 50,2$ & $144,8 \pm 3,4$ & $-10,8$ & 0,441 & $-39,1$ & 17,4 \\
\hline $\mathrm{R}$ & $39,2 \pm 13,4$ & $34,8 \pm 10,8$ & 4,4 & 0,268 & $-3,6$ & 12,4 \\
\hline
\end{tabular}

Teste t de Student para amostras independentes $(p<0,05)$

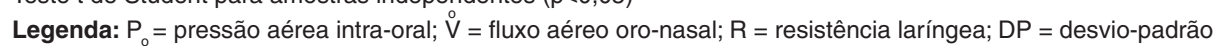

Tabela 5. Comparação entre os valores médios e desvio-padrão de pressão aérea intra-oral, fluxo aéreo laríngeo e resistência laríngea do grupo com fechamento velofaríngeo marginal com a oclusão das narinas com o grupo controle

\begin{tabular}{|c|c|c|c|c|c|c|}
\hline \multirow[t]{2}{*}{ Variáveis } & \multicolumn{2}{|c|}{ Média \pm DP } & \multirow[t]{2}{*}{ Diferença média } & \multirow[t]{2}{*}{ Valor de $p$} & \multicolumn{2}{|c|}{ Intervalo de confiança (95\%) } \\
\hline & Controle & Com oclusão & & & Limite inferior & Limite superior \\
\hline$P_{0}$ & $4,8 \pm 0,8$ & $4,8 \pm 1,1$ & 0,0 & 0,934 & $-0,7$ & 0,6 \\
\hline$\stackrel{\circ}{\mathrm{V}}$ & $133,9 \pm 50,2$ & $150,9 \pm 38,7$ & $-17,0$ & 0,251 & $-46,5$ & 12,5 \\
\hline $\mathrm{R}$ & $39,2 \pm 13,4$ & $34,0 \pm 14,3$ & 5,3 & 0,246 & $-3,8$ & 14,4 \\
\hline
\end{tabular}

Teste t de Student para amostras independentes $(p<0,05)$

Legenda: $P_{0}=$ pressão aérea intra-oral; $\stackrel{\circ}{V}=$ fluxo aéreo oro-nasal; $R$ = resistência laríngea; $D P=$ desvio-padrão

velofaríngea e laríngea no grupo de pacientes que apresentam alterações estruturais mínimas do fechamento velofaríngeo, condição denominada disfunção velofaríngea marginal.

Os resultados do presente estudo mostraram que, à seme- lhança de trabalhos anteriormente realizados no Laboratório de Fisiologia do HRAC-USP com pacientes que apresentavam disfunção velofaríngea grave ${ }^{(18-19)}$, a resistência laríngea de indivíduos com fissura de palato e disfunção velofaríngea 
marginal não diferiu da resistência laríngea de indivíduos sem fissura. Em parte, estes resultados concordam, também, com estudos que, utilizando metodologia similar ${ }^{(15)}$, não encontraram diferenças estatisticamente significantes entre pacientes com fissura e o grupo normal. Entretanto, estes autores sugeriram que a falta de significância estatística entre os seus resultados seria devida ao fato de que o grupo de indivíduos com fissura de palato apresentava fechamento velofaríngeo normal, não havendo, portanto, diferenças no fechamento velofaríngeo entre os dois grupos. Os autores discutiram, também, a extensa variação entre os parâmetros de $\mathrm{P}_{\mathrm{o}}, \stackrel{\circ}{\mathrm{V}}$ e R avaliados em ambos os grupos. Pode-se especular que tal variação seja decorrente da falta de controle e monitoramento da intensidade vocal ou do nível de pressão intra-oral utilizado pelo paciente, durante as emissões na avaliação da resistência laríngea, variáveis muito bem controladas no presente estudo. Uma vez que a resistência laríngea é diretamente proporcional à pressão aérea, variações no esforço vocal podem ocasionar variações na resistência laríngea em um mesmo indivíduo, o que pode ser evitado com o monitoramento da intensidade vocal.

Diferentemente do presente estudo, crianças com fissura labiopalatina e inadequação velofaríngea apresentaram maior resistência laríngea que crianças sem fissura e crianças com fissura e fechamento velofaríngeo completo, embora nenhuma diferença estatisticamente significante tenha sido encontra$\mathrm{da}^{(16)}$. No presente estudo, ao contrário, os pacientes com disfunção velofaríngea marginal tenderam a apresentar resistência laríngea menor (em média) que o grupo controle, o que foi, provavelmente, consequência do elevado valor de fluxo aéreo oro-nasal encontrado nestes pacientes. Outro aspecto importante também a ser observado é que, naquele estudo ${ }^{(16)}$, a avaliação da resistência laríngea foi realizada em duas condições. Em uma delas, os pacientes produziam as sílabas em intensidade vocal habitual, e em outra, os pacientes produziam as sílabas em intensidade vocal pré-determinada, utilizando um nível de pressão aérea intra-oral estabelecido pelo autor, monitorado visualmente pelo paciente e avaliador na tela do sistema. $\mathrm{O}$ autor verificou que, quando as medidas foram realizadas no nível de pressão intra-oral pré-determinado, os valores de resistência laríngea e de pressão aérea intra-oral diminuíram em relação àqueles obtidos durante a produção em intensidade habitual nos três grupos estudados, tornando-se mais semelhante entre os grupos. No presente estudo, o nível de pressão intra-oral também foi monitorado pelo avaliador; entretanto, o valor de pressão oral utilizado durante a medida da resistência laríngea foi pré-determinado pelo próprio paciente, a partir do valor de pressão oral obtido durante a repetição de uma frase produzida em intensidade vocal habitual e confortável.

Diferentemente dos trabalhos anteriores, alguns autores ${ }^{(17)}$ verificaram que pacientes com fissura labiopalatina e fechamento velofaríngeo adequado apresentaram resistência laríngea significativamente maior que os pacientes com disfunção velofaríngea grave. Este estudo levou os autores a concluírem que indivíduos que apresentam fechamento velofaríngeo marginal ou fechamento velofaríngeo completo, poderiam alcançar este fechamento velofaríngeo aumentando o esforço respiratório e consequentemente, a resistência laríngea, o que não seria possível para aqueles com disfunção velofaríngea grave. Os resultados do presente estudo, entretanto, não confirmaram essa hipótese. Curiosamente, ao contrário do que a literatura sugere, no presente estudo observou-se, individualmente, uma tendência do grupo com fissura em apresentar valores de resistência laríngea ligeiramente menores que dos indivíduos sem fissura. Uma vez que a resistência laríngea é inversamente proporcional ao fluxo oro-nasal, esta tendência a valores mais reduzidos pode ser explicada pela variação nos valores de fluxo oro-nasal, os quais foram maiores no grupo com fechamento marginal. Esta tendência a valores maiores de fluxo aéreo oro-nasal no grupo com fissura, foi também encontrada em um grupo com fechamento velofaríngeo incompleto ${ }^{(17)}$. Este achado pode ser explicado pela teoria que estabelece que, na presença de disfunção do mecanismo velofaríngeo, ocorrem ajustes fisiológicos como uma estratégia compensatória, com o objetivo de manter os níveis de pressão aérea intra-oral estáveis para a produção da fala, como o aumento do fluxo aéreo laríngeo ${ }^{(10)}$. Seguindo este raciocínio, estudos mostram que a diminuição da resistência em algum ponto do trato vocal resulta no aumento do fluxo aéreo necessário para manter os níveis estáveis de pressão aérea para a fala. Uma vez que a disfunção velofaríngea ocasiona o escape de ar pelo orifício velofaríngeo e, portanto, uma diminuição da resistência velar, o aumento do fluxo aéreo ocorre para que a aerodinâmica do trato vocal não se desestabilize ${ }^{(24)}$.

$O$ fato de não terem sido encontradas diferenças estatisticamente significantes entre a resistência laríngea de indivíduos com fechamento velofaríngeo marginal e indivíduos sem fissura no presente estudo, pode, ainda, estar relacionado à atividade muscular do mecanismo velofaríngeo desempenhada durante a produção da fala. Pacientes com fechamento velofaríngeo incompleto podem conseguir uma maior aproximação das estruturas velofaríngeas com o aumento do esforço respiratório somente durante uma emissão curta, como é o caso da sílaba. Durante a fala encadeada, onde há um aumento da velocidade da emissão, estes pacientes não conseguem manter o esforço respiratório aumentado e tendem a apresentar o fechamento velofaríngeo marginal e consequentemente a hipernasalida$\mathrm{de}^{(12)}$. Em suma, o fechamento velofaríngeo pode ser completo em palavras ou sílabas, mas é perdido durante a fala encadeada. Além disso, estes autores demonstraram que a fadiga do músculo levantador do palato é maior nos indivíduos com fechamento velofaríngeo marginal do que nos indivíduos sem fissura, decorrente, provavelmente, da atividade muscular desempenhada por aqueles indivíduos ser mais próxima do limite, portanto, mais intensa. A diferença no fechamento velofaríngeo entre sílabas e frases em paciente com disfunção velofaríngea já foi demonstrada em estudo realizado no Laboratório de Fisiologia do HRAC-USP ${ }^{(25)}$ no qual verificou-se que $67 \%$ dos pacientes com fissura labiopalatina e DVF conseguiram fechamento velofaríngeo adequado na emissão de sílabas, e uma menor proporção de $52 \%$, na produção de frases. Esta pode ser a explicação para nossos resultados. No presente estudo, uma vez que se pretendeu determinar a resistência laríngea empregada habitualmente, foi utilizada uma frase para se conhecer o nível de pressão oral e, consequentemente o esforço que o paciente utiliza durante a fala. As medidas da resistência laríngea foram, então, realizadas utilizando-se esta 
pressão intra-oral habitual como valor de referência, critério este que não foi utilizado em nenhum dos estudos citados. Verificou-se que a pressão intra-oral dos pacientes não foi diferente da pressão intra-oral dos indivíduos do grupo controle, ou seja, os pacientes com fechamento velofaríngeo marginal estudados não aumentaram o nível de pressão oral quando solicitados a produzir a fala numa intensidade de voz habitual. Utilizando a linha de raciocínio proposta pela literatura ${ }^{(11-12)}$, isto seria esperado uma vez que um maior esforço respiratório não poderia ser mantido durante a conversação espontânea ao longo do dia. Como durante a avaliação da resistência laríngea, a produção da sílaba "pa" foi monitorada a fim de se manter o mesmo nível de pressão intra-oral habitual, é possível que, se tal controle não tivesse sido feito, os pacientes com fissura aumentassem a intensidade e esforço para melhorar a ressonância, aumentando, consequentemente a resistência laríngea. A possibilidade disto ter ocorrido naqueles estudos ${ }^{(15-18)}$ que demonstraram uma tendência dos pacientes com fissura labiopalatina apresentarem resistência laríngea maior que o normal deve ser considerada. É possível que estes pacientes aumentem a resistência laríngea, na tentativa de alcançar o fechamento velofaríngeo adequado, durante emissões curtas, o que não significa que esta manobra ocorra na conversa espontânea a ponto de modificar a aerodinâmica da laringe.
Sendo assim, apesar do fechamento velofaríngeo marginal representar um desafio para os clínicos devido a suas características fisiológicas singulares, as quais acarretam alterações de fala muitas vezes perceptíveis, os resultados deste estudo mostraram que, do ponto de vista aerodinâmico, a disfunção velofaríngea marginal não interferiu no comportamento da laringe.

\section{CONCLUSÃO}

Os pacientes com fissura labiopalatina e fechamento velofaríngeo marginal estudados não modificaram a resistência laríngea, contrariando a hipótese de que tais pacientes poderiam aumentar o esforço respiratório e vocal numa tentativa de alcançar o fechamento velofaríngeo completo. Além disso, a oclusão das narinas, condição que elimina o escape de ar pelo nariz, não modificou a resistência laríngea nos indivíduos com fechamento velofaríngeo marginal estudados.

\section{AGRADECIMENTOS}

À Coordenação de Aperfeiçoamento de Pessoal de Nível Superior (CAPES), pelo apoio financeiro concedido para esta pesquisa.

\begin{abstract}
Purpose: To verify whether patients with marginal velopharyngeal dysfunction modify the laryngeal resistance as a strategy to achieve complete velopharyngeal closure during speech. Methods: Nineteen patients of both genders with repaired cleft palate with marginal velopharyngeal closure and ages varying from 12 to 47 years, and 18 patients of both genders without cleft palate (control group), with ages between 14 and 35 years were assessed. Laryngeal resistance $(\mathrm{R})$, intraoral air pressure $\left(\mathrm{P}_{\mathrm{o}}\right)$ and the oronasal airflow $\left(\mathrm{V}^{\circ}\right)$ were obtained through an aerodynamics evaluation using the PERCI-SARS system during the production of the syllable /pa/, with and without nasal occlusion. Results: Mean values of laryngeal resistance $(\mathrm{R})$, intraoral air pressure $\left(\mathrm{P}_{\mathrm{o}}\right)$ and oronasal airflow $(\stackrel{\circ}{\mathrm{V}})$, for the group with marginal velopharyngeal dysfunction, were, respectively, $34.8 \pm 10.8 \mathrm{cmH}_{2} \mathrm{O} / \mathrm{L} / \mathrm{sec}, 4.8 \pm 1.4 \mathrm{cmH} \mathrm{O}_{2}, 144.8 \pm 34.0$ $\mathrm{mL} / \mathrm{s}$ without nasal occlusion, and $34.0 \pm 14.3 \mathrm{cmH}_{2} \mathrm{O} / \mathrm{L} / \mathrm{sec}, 4.8 \pm 1.1 \mathrm{cmH}_{2} \mathrm{O}, 150.9 \pm 38.7 \mathrm{~mL} / \mathrm{s}$ with nasal occlusion. In the control group, the mean values were $39.2 \pm 13.4 \mathrm{cmH}_{2} \mathrm{O} / \mathrm{L} / \mathrm{sec}, 4.8 \pm 0.8 \mathrm{cmH}_{2} \mathrm{O}, 133.9 \pm 50.2 \mathrm{~mL} / \mathrm{s}$, respectively, for the same variables. There was no statistically significant difference $(\mathrm{p}<0.05)$ between the groups regarding mean values of laryngeal resistance $(\mathrm{R})$, intraoral air pressure $\left(\mathrm{P}_{\mathrm{o}}\right)$ and oronasal airflow $(\stackrel{\mathrm{V}}{\mathrm{V}})$. Conclusion: These results showed that the patients with marginal velopharyngeal closure studied did not modify laryngeal resistance.
\end{abstract}

Keywords: Velopharyngeal insufficiency; Cleft palate; Larynx; Speech; Voice

\title{
REFERÊNCIAS
}

1. Zuiani TB, Trindade IE, Yamashita RP, Trindade AS Jr. The pharyngeal flap surgery in patients with velopharyngeal insufficiency: perceptual and nasometric speech assessment. Braz J Dysmorphol Speech Disord. 1998;2(1):31-42.

2. Smith BE, Kuehn DP. Speech evaluation of velopharyngeal dysfunction. J Craniofac Surg. 2007;18(2):251-61.

3. Peterson-Falzone SJ, Hardim-Jones MA, Karnell MP. Cleft palate speech. Saint Lowis: Mosby; 2001. Implications of inadequate velopharyngeal function for articulation, resonance and voice; p. 273-88.

4. Brown AS, Cohen MA, Randall P. Levator muscle reconstruction: does it make a difference? Plast Reconstr Surg. 1983;72(1):1-8.
5. Dumbach J. Refinements of intravelar veloplasty. Scand J Plast Reconstr Surg Hand Surg. 1987;21(1):103-7.

6. Billmire DA. Surgical management of clefts and velopharyngeal dysfunction. In: Kummer AW. Cleft palate and craniofacial anomalies: the effects on speech and resonance. San Diego: Singular; 2001. p.40124.

7. Trindade IE, Genaro KF, Yamashita RP, Miguel HC, Fukushiro AP. Proposta de classificação da função velofaríngea na avaliação perceptivo-auditiva da fala. Pró-Fono. 2005;17(2):259-62.

8. Sie KC, Tampakopoulou DA, Sorom J, Gruss JS Eblen LE. Results with Furlow palatoplasty in management of velopharyngeal insufficiency. Plast Reconstr Surg. 2001;108(1):17-25. 
9. Penido FA, Noronha RM, Caetano KI, Jesus MS, Di Ninno CQ, Britto AT. Correlação entre os achados do teste de emissão de ar nasal e da nasofaringoscopia em pacientes com fissura labiopalatina operada. Rev Soc Bras Fonoaudiol. 2007;12(2):126-34.

10. Warren DW. Compensatory speech behaviors in individuals with cleft palate: a regulation/control phenomenon? Cleft Palate J. 1986;23(4):251-60.

11. Kummer AW. Cleft palate and craniofacial anomalies: the effects on speech and resonance. San Diego: Singular; 2001. Velopharyngeal dysfunction (VPD) and resonance disorders; p.145-76.

12. Nohara K, Tachimura T, Wada T. Levator veli palatini muscle fatigue during phonation in speakers with cleft palate with borderline velopharyngeal incompetence. Cleft Palate Craniofac J. 2006;43(1):1037.

13. Tachimura T, Nohara K, Satoh K, Wada T. Evaluation of fatigability of the levator veli palatini muscle during continuous blowing using power spectra analysis. Cleft Palate Craniofac J. 2004;41(3):320-6.

14. Netssel R, Lotz W, Shaughnessy AL. Laryngeal aerodynamics associated with selected voice disorders. Am J Otolaryngol. 1984;5(6):397-403.

15. Lewis JR, Andreassen ML, Leeper HA, Macrae DL, Thomas J. Vocal characteristics of children with cleft lip/palate and associated velopharyngeal incompetence. J Otolaryngol. 1993;22(2):113-7.

16. Zajac DJ. Laryngeal airway resistance in children with cleft palate and adequate velopharyngeal function. Cleft Palate Craniofac J. 1995;32(2):138-44.

17. Guyette TW, Sanches AJ, Smith BE. Laryngeal airway resistance in cleft palate children with complete and incomplete velopharyngeal closure. Cleft Palate Craniofac J. 2000;37(1):61-4.
18. Yamashita RP, Trindade IE, Louzado MR, Miguel HC, Trindade Júnior AS. Resistência laríngea em indivíduos normais e em portadores de insuficiência velofaríngea. Acta AWHO. 2000;19(1):10-7.

19. Yamashita RP, Calais LL, Miguel HC, Trindade IE. Avaliação da resistência laríngea em indivíduos portadores de insuficiência velofaríngea com distúrbio articulatório compensatório. Acta ORL. 2006;24(4):263-7.

20. Genaro KF, Yamashita RP, Trindade IE. Avaliação clínica e instrumental na fissura labiopalatina. In: Ferreira LP, Befi-Lopes DM, Limongi SC. Tratado de fonoaudiologia. São Paulo: Roca; 2004. p. 456-77.

21. Hirano M. Clinical examination of voice. New York: Springer-Verlag; 1981.

22. Smitheran JR, Hixon TJ. A clinical method for estimating laryngeal airway resistance during vowel production. J Speech Dis. 1981;46(2):138-46.

23. Morettin PA, Bussab WO. Estatística básica. 6a ed. São Paulo: Saraiva; 2006.

24. Laine T, Warren DW, Dalston RM, Morr KE. Effects of velar resistance on speech aerodynamics. Eur J Orthod. 1989;11(1):52-8.

25. Fukushiro AP, Yamashita RP, Sampaio AC, Trindade IE. Velopharyngeal closure following pharyngeal flap surgery assessed by pressure-flow technique. In: $10^{\text {th }}$ International Congress on Cleft Palate and Related Craniofacial Anomalies; 2005 4-8 Sept; Durban, South Africa. Final programme \& book of abstracts . p.202-3. 